\title{
Generalization and Differentiation in Semantic Memory
}

\author{
Insights from Semantic Dementia
}

\author{
Matthew A. Lambon RalPh ${ }^{a}$ ANd KaRalyn Patterson ${ }^{b}$ \\ ${ }^{a}$ Neuroscience and Aphasia Research Unit, School of Psychological Sciences, \\ University of Manchester, Manchester, United Kingdom \\ ${ }^{b}$ MRC Cognition and Brain Sciences Unit, Cambridge, United Kingdom
}

\begin{abstract}
According to many theories, semantic representations reflect the parallel activation of information coded across a distributed set of modality-specific association brain cortices. This view is challenged by the neurodegenerative condition known as semantic dementia (SD), in which relatively circumscribed, bilateral atrophy of the anterior temporal lobes results in selective degradation of core semantic knowledge, affecting all types of concept, irrespective of the modality of testing. Research on SD suggests a major revision in our understanding of the neural basis of semantic memory. Specifically, it is proposed that the anterior temporal lobes form amodal semantic representations through the distillation of the multimodal information that is projected to this region from the modality-specific association cortices. Although cross-indexing of modality-specific information could be achieved by a web of direct connections between pairs of these regions, amodal semantic representations enable semantic generalization and inference on the basis of conceptual structure rather than modality-specific features. As expected from this hypothesis, SD is characterized by impaired semantic generalization, both clinically and in formal assessment. The article describes a comprehensive array of under- and overgeneralization errors by patients with SD when engaged in receptive and expressive verbal and nonverbal tasks and everyday behaviors.
\end{abstract}

Key words: semantic dementia; semantic memory; anterior temporal lobes

\section{Introduction}

The British poet Philip Larkin once famously quipped that sexual intercourse began in 1963, between the end of the Lady Chatterley ban and the arrival of the Beatles' first LP. Just as remarkably, though perhaps less sensationally, semantic memory appears to have begun around the same time. The first documented use of the term is probably the title of Quillian's (1966) Ph.D. thesis; it subsequently appeared as part of the title of the well-known article by Collins and Quillian (1969) and, even more famously, in Tulving's (1972) chapter on the distinction between episodic and semantic memory.

Of course, even before the 1960s, everyone really knew that people had semantic memory (as well as

\footnotetext{
Address for correspondence: Prof. Matthew A. Lambon Ralph, Neuroscience and Aphasia Research Unit, School of Psychological Sciences, University of Manchester, Manchester, UK.

matt.lambon-ralph@manchester.ac.uk
}

sex), but it is perhaps not so surprising that semantic memory has only recently achieved independent status. What we mean by having independent status is the equivalent of what Shallice (1988) refers to as a cognitive subsystem that is isolable or anatomically distinct. Semantic memory is usually assumed not to be localized to one neuroanatomical region but rather to reflect activation of modality-specific information encoded within a widely distributed network of regions. This view is perhaps most often found in the discussion sections of articles on functional imaging of semantic processing in normal individuals (e.g., Thompson-Schill 2003), though it is also sometimes found in studies of neuropsychological disorders. Here is one example. Some objects, such as elephants or spinach, have characteristic colors, and all cognitive scientists would probably agree that people's knowledge of these standard colors is part of semantic memory. But suppose, as some researchers have concluded in their imaging or patient studies, that color knowledge has its own module in the brain, separate from other aspects of knowledge even about the same objects (e.g., what shape elephants are, 
what sound they make, and how they move). Then color knowledge would have independent status and a specific neuroanatomical location, and so would each of the other attribute-specific components of semantic memory (Martin 2007); but central conceptual knowledge would not have this characteristic.

In this article we will outline a different hypothesis about semantic memory, in which - in addition to the modality-specific components of the broader semantic network on which essentially all theories agree - the central aspects of conceptual knowledge are and in fact need to be synthesized into a localized subsystem of unified, amodal representations. More specifically, the hypothesis comes in two parts: (a) as conceptual knowledge is acquired, the brain constructs amodal semantic representations that knit together the collection of attribute-specific features about a concept; and (b) neuroanatomically speaking, these amodal representations are supported by anterior regions of the temporal lobes bilaterally. Why do we claim that central semantic memory ought to be localized in this fashion? The argument, developed below, is that amodal conceptual representations support the vital function of semantic generalization. The corollary of this argument is that, when the brain region supporting these representations is damaged, a key consequence will be a failure of appropriate generalization.

\section{Conceptualization without Amodal Representations}

Before laying out the theory and evidence for amodal semantic representations, we will first briefly review both historical and contemporary views of semantic memory. Long before the term "semantic memory" was introduced to the world, in fact going back more than a century, Wernicke and Meynert (see Eggert 1977) were interested in how the brain formed and reactivated concepts - a process they referred to as "conceptualization." Unlike the language centers that Wernicke, more famously, described in his work on aphasia, Meynert and Wernicke's model of conceptualization made the following assumptions: (a) that the building blocks of concepts were modality-specific engrams (stores of information) localized to the cortical areas responsible for the corresponding sensory, motor, or verbal domain; (b) that these modality-specific engrams, in widespread brain regions, were fully interconnected; and (c) that this web of connections was the basis of conceptualization - a specific concept being represented by the coactivation of all its associated engrams (FIG. 1A). For example, if you taste an apple (even with your eyes closed), the taste-specific engram will automatically activate all the other asso- ciated modality-linked engrams, enabling your brain to retrieve other knowledge concerning the object: its visual form, probable color, name, presence of seeds, how you would peel it, and so on. In this proposal, modality-specific engrams were located in particular brain regions, but conceptualization was not. Indeed, Wernicke and Meynert argued that, unlike forms of agnosia and aphasia, central disorders of conceptualization occurred only as a consequence of global brain damage (dementia) because only such widespread cortical damage would disrupt the engram reactivation process.

\section{Conceptualization Requires Amodal Representations}

In this article we advance the hypothesis that semantic memory is, in fact, critically based on the formation of amodal representations, and we attempt to explain why this form of semantic organization might be required for the complexities of conceptual behaviour in which humans specialize. The first significant modern evidence for this hypothesis came in the form of a neuropsychological study by Warrington (1975). She described three patients with progressive brain disease resulting in a range of semantic deficits that cut across different modalities but were nonetheless restricted to the domain of semantics. That is, other aspects of the patients' cognition, including perceptual abilities and even other forms of memory (everyday episodic memory and short-term memory) were well preserved. Referring to Tulving's (1972) then-recently proposed distinction between episodic and semantic memory, Warrington (1975) labeled the disorder one of semantic memory. Snowden et al. (1989) later identified the condition as part of the spectrum of frontotemporal dementia (FTD) and gave it the label that has stuck, semantic dementia (SD).

There was little or no neuroanatomical information about the patients in Warrington's original study, but as further similar cases were published in tandem with advances in structural brain imaging, it became clear that SD was consistently associated with selective atrophy of the anterior temporal lobes bilaterally (Hodges et al. 1992; Snowden et al. 1996). Quantitative structural magnetic resonance imaging (MRI) studies, using both manual methods and automatic voxel-based morphometry, reveal consistent and substantial atrophy (often $50-80 \%$ gray matter loss) of the polar and perirhinal cortices and the anterior fusiform gyri (Williams et al. 2005; Brambati et al. 2007). In a neurodegenerative condition, precise boundaries of the pathological process are admittedly not easy to define; but, with the advent of functional brain imaging, 

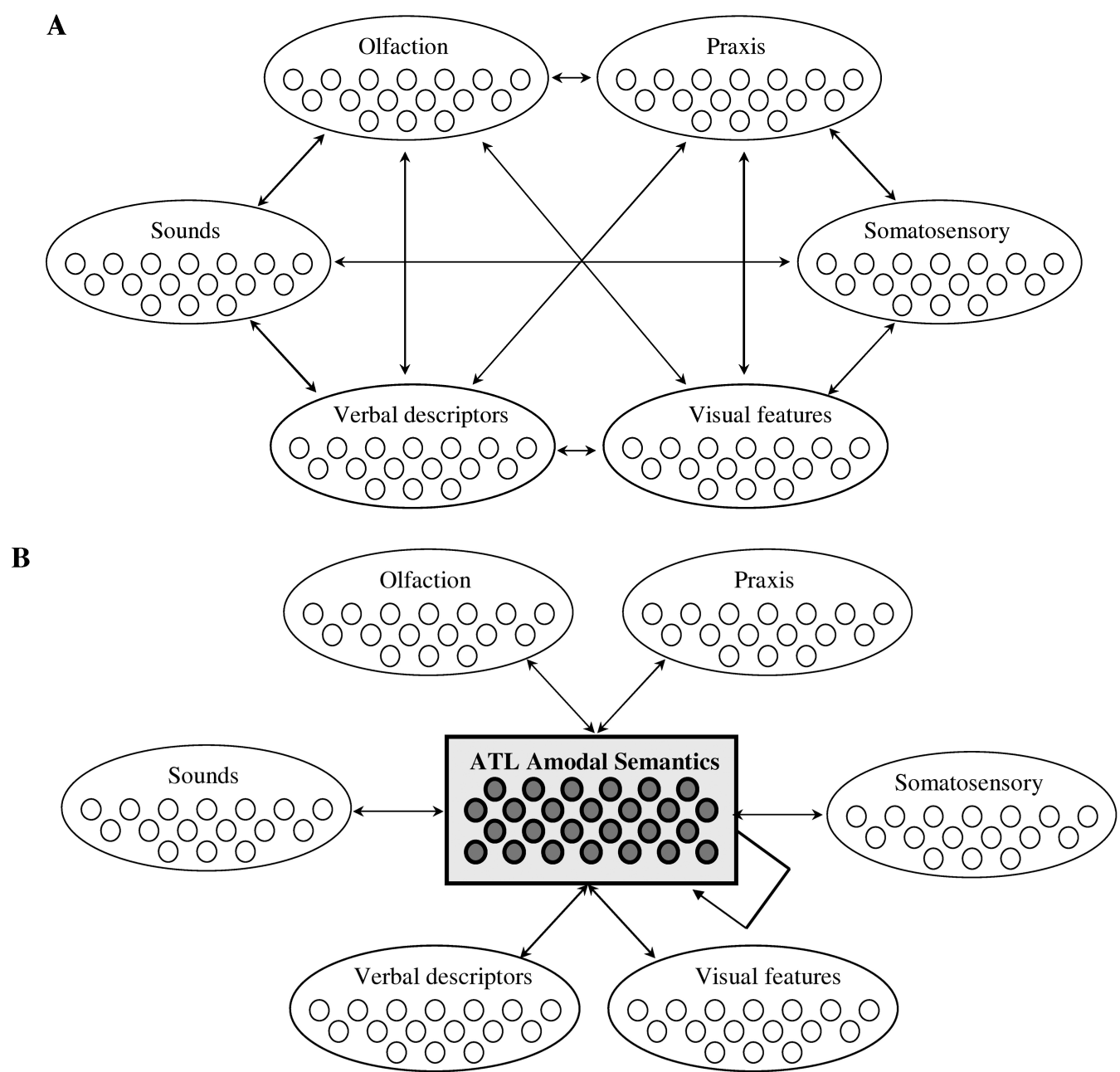

\section{KEY \\ $\bigcirc$ Modality-specific association areas ATL Amodal Semantic System}

FIGURE 1. (A) Wernicke-Meynert model of conceptualization. (B) Model of ATL amodal semantic system (Rogers et al. 2004).

researchers have been able to demonstrate that not only the atrophy but also the hypometabolism in SD is restricted largely to the anterior temporal lobes, suggesting that the associated cognitive disorder is attributable to damage in this region rather than to widespread functional abnormality (Desgranges et al. in press; Diehl et al. 2004; Nestor et al. 2006).

While patients are alive, the nature of the dysfunction in neurodegenerative disease can only be guessed at or predicted, albeit often (and increasingly) with reasonably high confidence. Confirmed pathological diag- nosis requires postmortem analysis of brain tissue, and researchers studying FTD in general and SD in particular have only recently begun to accumulate sufficient postmortem samples to be able to offer meaningful summary statistics. In patients coming to postmortem from our Cambridge series, of 20 cases with a clinical diagnosis of SD in life, 14 had ubiquitin-positive neuronal inclusions of the kind associated with motor neuron disease; four had the $\tau$-positive inclusions characteristic of Pick's disease; and two had the plaques and tangles of Alzheimer's disease. 
As well as data on the regions of atrophy and underlying dysfunction in SD, there is a considerable wealth of detailed neuropsychological findings about this disorder (Rogers et al. 2004; Patterson et al. 2007; Hodges $\&$ Patterson 2007). In short, the patients present with a progressive and selective deterioration of semantic memory. Progressive means that, especially if the patients come to appropriate assessment early enough, their performance on tests requiring conceptual knowledge can be tracked from mildly to moderately to severely abnormal; selective means that many other aspects of cognition, memory, and language are relatively well preserved until late in the disorder. This specificity is important for several reasons. Clinically, it is an important aspect for differential diagnosis of SD as opposed to other forms of dementia or conditions in which semantic impairment can be observed (Hodges \& Patterson 2007; Kipps et al. 2008). From a research perspective, the specificity of the semantic decline means that SD provides a neurological model of semantic impairment and its effect on cognition without major complications from co-occurring nonsemantic deficits. Of course, semantic impairment can be found in several other neurological conditions, including Alzheimer's disease, herpes simplex virus encephalitis, head injury, and stroke. Almost inevitably, however, the semantic impairment in these disorders is (a) less pervasive than that observed in SD and (b) accompanied by other deficits affecting episodic or shortterm memory, attention, executive, and/or language processing.

SD challenges the assumption that semantic memory is based on a widely distributed system of modalityspecific representations and links between them and does not require amodal representations localized to a specific brain region. For this article two more characteristics of SD are important: (a) semantic impairment is a graded phenomenon in which concepts and the boundaries between concepts gradually "dissolve" or "dim," rather than dropping out abruptly (Lambon Ralph et al. 2007; Patterson \& Hodges 2000); (b) the degradation of concepts is amodal, thereby producing poor comprehension and production of information across all verbal and nonverbal domains, including words, objects, pictures, sounds, smells, and touch, etc., in receptive mode, and speech, writing, object use, drawing, etc., in expressive mode (Rogers et al. 2004).

These characteristics of SD can be understood on the hypothesis that the area of focal atrophy in this disorder, the anterior temporal lobe (ATL), supports the formation of amodal semantic representations. Using a computational model, Rogers et al. (2004) demon- strated that a central component of the model's architecture that draws together modality-specific information will behave as an amodal semantic system. The Rogers et al. model is an extension of the MeynertWernicke framework (FIG. 1B). Information arising in each specific modality (e.g., the elephant's shape, color, smell, form of movement, name, and verbal descriptors) is coded in the corresponding specific cortical sensory or motor or language region; in this sense, the semantic network as a whole is neuroanatomically widespread. The information from the modalityspecific regions, however, is fused through amodal representations. We assume these representations to be supported by the bilateral ATL, not only because this is the location of the focal atrophy/hypometabolism in SD but also because this region is highly interconnected with many modality-specific association cortices (Gloor 1997); it thus has the necessary neuroanatomical connectivity to provide the neural substrate for the fusion of multimodal information into amodal representations. The Rogers et al. model was trained to take a piece of modality-specific information (e.g., an outline of the elephant's visual form) as input and to reproduce the correct information across the remaining information layers (e.g., its color or name or various things people might say about it) by propagating activation through the intermediate units. Rogers et al. demonstrated that simulated damage to these intermediate semantic units of the trained model reproduced the core features of SD. That is, increasing degrees of damage produced gradual decline in performance of any task requiring conceptual knowledge and, because of its intermediate location, damage to this central component of semantic memory resulted in deficits across various receptive and productive verbal and nonverbal domains.

At this level of description, the implemented Rogers model provides an alternative to the WernickeMeynert solution to the riddle of conceptualization (how to draw together multimodal experience, coded in different brain regions, into a unified concept). The Rogers formulation also resembles the notion of "convergence zones" proposed by Damasio (1989), whereby various brain regions link or index corresponding features and attributes. Logically speaking, conceptualization could be achieved either by the full set of direct connections between individual pairs of modalityspecific regions (Wernicke-Meynert) or by a common amodal portal (Rogers/Damasio et al.). Semantic dementia, however, in which a selective but multimodal semantic impairment results from circumscribed ATL damage, argues for the second solution. If this assertion 
is correct, one might want to inquire why nature has selected against what might seem the simpler brainengineering solution (a web of direct connections) and instead used a corner of the brain (the ATL) to underpin conceptualization. The answer, or at least one potential answer, lies in generalization. Knowing the range of concepts over which a component of knowledge should be generalized is a multilevel, nonlinear problem; its solution probably requires representations that abstract away from surface similarities.

\section{The Conundrum of Semantic Generalization and Inference}

The following example captures critical aspects of the semantic conundrum of generalization and inference. Various exemplars of five different yet related objects are shown in FigURE 2. The items are grouped horizontally in terms of visual similarity, whereas the vertical structure reflects their most typical function. One can immediately see that superficial, surface similarity is not a reliable guide to other types of information. Thus, to know which subset of objects would be used to make and serve tea (a nonverbal generalization) or be referred to as a "teapot" (a verbal generalization) requires us to step away from visual similarities to select the correct subset. Matters are made more complex because different pieces of verbal or nonverbal knowledge can be generalized at different levels across this set. For example, all the items are used to contain and pour liquids (and we might refer to them verbally as "containers" to denote this level of generality). Consequently, they need to be set down on a level surface, and the liquid level needs to be kept below the tip of the spout to avoid spillage. The exact form of the liquid, however, varies across these objects - one set takes oil, one group takes wine, whereas the others hold water. Then at a more specific level, two of the five are used to hold very hot water (yet we do not use the same name to refer to them), but only one of these is used to generate it. Each of the five main groups of items can be subdivided again in terms of both the verbal and nonverbal features applied to them. For example, some of the kettles are heated by being set on a flame or external electric element, whereas others incorporate an internal electric element and so are plugged in (a distinction that can confuse British and American people when they visit each other's houses). A subset of teapots are made of metal or china (the latter therefore fragile), but the teapots can also be divided along completely different lines in terms of those that would be used to serve "English" versus Chinese or Japanese tea. Finally, we can use our complex semantic knowledge of these items to make new inferences on the basis of their meaning rather than their visual or structural characteristics. For example, if we learn about a new type of tea or learn that the Japanese word for teapot is kyusu, we would all pick the same subset of items to infuse the new tea or to apply the new label to- and we would do so on the basis of conceptual rather than visual similarity.

In summary, an amodal semantic system performs five key yet computationally demanding functions: (a) it provides a method to link multimodal experience and information to a core concept; (b) it allows us to step away from surface similarities (e.g., similar-sounding or -looking objects) to generalize on the basis of conceptual similarity; (c) it permits generalization of features simultaneously at different levels of specificity; (d) it allows appropriate generalization to be computed for all types of verbal and nonverbal information; and (e) it provides a sophisticated knowledge base on which to make new inferences when we acquire a new piece of information.

\section{SD: A Disorder of Failing Semantic Generalization?}

If the neuroanatomical basis of amodal semantic representations is the ATL, patients with SD in whom focal brain disease gradually destroys the ATL - should exhibit disorders in all five functions listed above. To date, research on SD has concentrated largely on the first of these - the ability to link verbal and nonverbal information to each concept. As expected, disease progression in SD leads to a striking and eventually profound disruption of semantic knowledge. Thus, the patients have significant deficits in understanding and producing the names of objects (Hodges et al. 1992; Warrington 1975), provide increasingly impoverished definitions and drawings of objects (Lambon Ralph et al. 1999), have a reducing repertoire of everyday behavior such as knowing how to cook or cut the lawn or replace a fuse (Hodges \& Patterson 2007), and so on. More recently, however, researchers studying SD have begun to investigate the other four, arguably more complex, aspects of conceptualization (e.g., Patterson 2007). The next section of this article will review and synthesize current evidence for disorders of these functions in SD, drawing upon evidence from both formal neuropsychological assessment and from everyday, real-world examples. Specifically, by assessing the effect of ATL degeneration in SD, we will evaluate the hypothesis that the ATL semantic system provides the mechanism for appropriate generalization on the basis of amodal, conceptual knowledge 


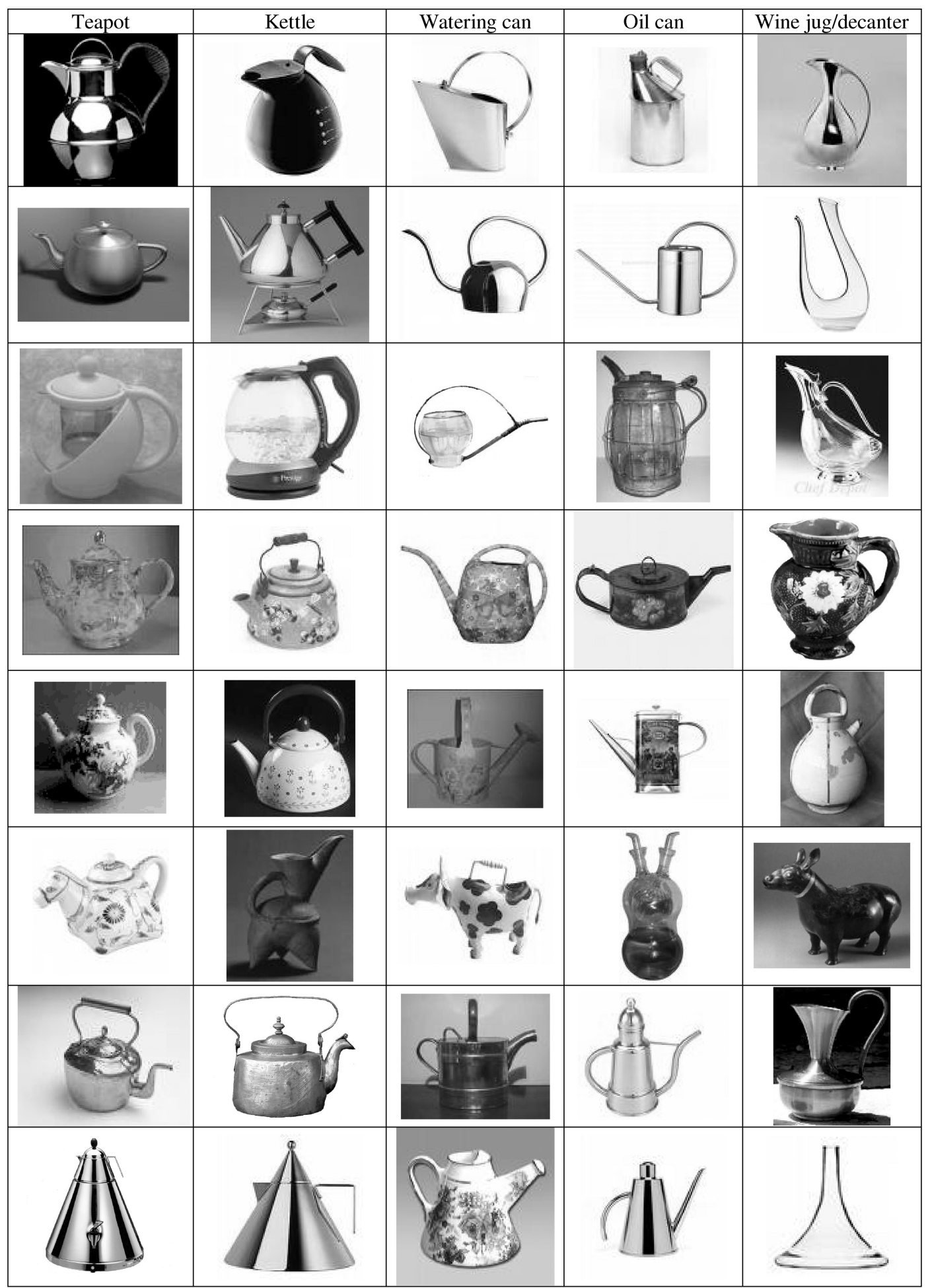

FIGURE 2. Examples of "liquid containers" ordered by visual similarity (rows) and principal function (columns). 
(i.e., not on the basis of modality-specific similarities). If this notion is correct, then we should be able to find evidence for the following three predictions:

(a) Patients with SD should both over- and undergeneralize knowledge, being increasingly influenced by information that is general to many items rather than specific to a given concept, and by superficial, nonsemantic similarities.

(b) They should exhibit over- and undergeneralization in both verbal and nonverbal domains.

(c) They should exhibit over- and undergeneralization in both receptive and expressive tasks.

\section{Evidence from SD Regarding the Nature of Under- and Overgeneralization}

\section{Word Production and Comprehension}

The first - and throughout the course of the disease, perhaps always most obvious - symptom of SD is anomia: inability to retrieve the appropriate word for an object or concept. This anomia should not be thought of as word-finding difficulty in the sense experienced by many patients with aphasia after a stroke, or indeed sometimes momentarily by normal people. In the latter cases, people will often spontaneously retrieve the desired word a few minutes later or on another occasion; and even when in the anomic state they can often be helped to retrieve it by a simple cue such as its initial letter or sound (Jefferies \& Lambon Ralph 2006). The anomia in SD is neither temporary nor cueable, and for good reason: The source of SD anomia is not in fact a failure of word retrieval. Its cause is that the patients lack sufficiently precise knowledge of the concept for the semantic system to activate the concept's name. Cueing (e.g., for violin, "it begins with /v/") will help normal speakers and most patients who suffered an aphasic stroke because they have substantial semantic information about the concept; the experimenter's additional bit of phonological information $(/ \mathrm{v} /)$ can then push the word's phonological representation over the threshold for production. Patients with mild SD, looking at a picture of a violin, know only that it is a musical thing; patients with more severe SD may not know what it is at all. This degraded information is insufficient for them to find the word on another occasion or to benefit from a phonological cue (Graham et al. 1995).

Given that the anomia in SD derives from degraded knowledge, anomia is naturally accompanied by a parallel deficit in word comprehension. Asked "What is a violin?" the patients' responses mimic their attempts to name a picture of it: That is, at a mild stage, the patient may say that "It's for playing music"; at a more severe stage, he or she will just say "I don't know." Indeed, in clinic we often observe something like "word alienation," where a patient responds by repeating "violin, violin, violin" over and over again, with perfect phonological production but no apparent experience of familiarity with the word or its referent (Hodges \& Patterson 2007).

How do the phenomena of under- and overgeneralization reveal themselves in the word production and comprehension deficits of patients with SD? For production, there are three sources of evidence: formal object-naming tests, formal analyses of target word production in a task like picture description, and anecdotal observations of the things that patients say in conversation or in clinic interview.

On naming tests, one prominent form of overgeneralization in SD is the production of semantic coordinate errors in which the name produced is a more common, more typical instance of the category to which the target instance belongs. This error occurs most often in the domain of animals because the category structure of animals lends itself most naturally to this kind of error. Hence the most common coordinate errors are the most typical and most familiar animal names ("cat," "dog," and "horse" in Britain). One additional intriguing component of this phenomenon is that, even though the line drawings usually used in these naming tests are all roughly the same size, the patients' semantic errors can indicate some residual knowledge of real-world size, with an elephant named as "horse," a goat named as "dog," and a rabbit named as "cat" (Hodges et al. 1995; Patterson et al. 2007). Another component of interest, and more directly relevant to the nature of overgeneralization, is that semantic coordinate errors (a) occur more often for moderately typical instances of a category than for atypical ones and (b) are more likely to be the prototypical dog-cat-horse responses if the target itself is fairly typical. In a recent large analysis of naming in patients with SD, for example, Woollams et al. (2007) reported on the fate of the tortoise and the hare (or rabbit), the former being an atypical animal and the latter a fairly typical one as indicated by category typicality norms (Morrow \& Duffy 2005). Of incorrect responses to these two pictures, a significantly higher proportion were semantic coordinate errors for the rabbit than for the tortoise; and of the semantic coordinate errors, a much higher proportion were "cat" or "dog" for the rabbit than for the tortoise. Our way of thinking about this phenomenon 
is that the amodal semantic representations for typical instances with many shared features are clustered together at the center of semantic space. When a patient's conceptual representation of rabbit is too degraded to generate the correct name, the brain activation produced by looking at a rabbit can be captured by a more intact and close concept like "cat" or "dog." "Tortoise," on the other hand, is far from the center of this space and so is less prone to being overgeneralized to the prototypical animals (Garrard et al. 2001; Woollams et al. 2007).

The other main form of overgeneralization in naming by patients with SD is production of a superordinate label such as "animal." Again, this type of error is more commonly observed for living things than for synthetic artifacts, though sometimes patients will use an artifact superordinate like "container" for objects that can hold things, such as cups, bowls, dustbins, suitcases, boxes, or bottles.

The results of tests like picture description tell a similar story, indicating that the profound anomia and overgeneralization observed in SD is not related to the perhaps odd and out-of-context experience of being asked to name a series of single, unrelated objects. In the picture-description task most often used in the aphasia literature, the Cookie Theft picture from Goodglass and Kaplan (1983), a woman is washing dishes at an overflowing sink, while behind and unseen by her, her son (presumably) is standing on a tipping stool as he tries to steal cookies from a cupboard. Almost every normal speaker, in describing this picture, uses the terms "stool" for what the boy is standing on and starting to fall off of, "sink" for what the woman is standing at, and "overflowing" for what is happening to the water in the sink. Of 21 patients with SD (whose descriptions of this picture were analyzed by Patterson \& MacDonald 2006), none produced the word "stool"; three managed to say "sink"; and two, "overflowing." Most patients, though not all, did produce a verb to describe what was happening to the water, but it was usually a more general term than "overflowing," such as "coming down" or "running out."

In spontaneous conversation, speech of patients with SD is replete with overgeneralizations, though of course they often just say little and so do not give researchers an opportunity to observe their word production failures - which is, of course, partly why we resort to tests like object naming or picture description. Virtually all the patients overuse terms like "thing" and "place" in conversation to substitute for the objects and locations that they cannot name. One patient began to call everyone of relevance in her life (e.g., her sis- ters, her son) "my person." Individual patients, as their semantic deficit worsens, often seem to alight on particular words to serve as general terms, and these are sometimes terms such as "situation" or "portion" that give the patient's speech an odd and almost formalsounding quality.

Overgeneralization is somewhat harder to observe in comprehension than production, in part because of the major difference in the nature of the mapping from words versus objects (or pictures of them) to concepts. A simple experiment, or even "thought" experiment, of removing one part of a stimulus easily illustrates this concept. If a picture of a duck is presented minus one component such as its head, feet, or tail, an observer is still likely to be able to identify it as a duck, or at least as a bird. By contrast, if we remove one letter from the written name of this object:_uck could be duck or luck; $d \_c k$ could be duck or dock; du_k could be duck or dusk; and duc_could be duck or duct. There is no "duckiness" about any of these alternative words. Despite the limited ability to infer anything about a word's meaning from its phonological or orthographic shape, at least early in the semantic deterioration of SD, patients will sometimes, as indicated above, comprehend a spoken word in an overgeneral fashion, as in "What's a violin?" $\rightarrow$ "A musical thing." One can also observe something akin to overgeneralization in the patients' word comprehension by constructing word-to-picture matching tests in which the semantic proximity of the target to the foils is manipulated. Patients with SD achieve reasonable (though certainly below normal) scores in pointing to a picture of a kingfisher in response to its spoken name when the foils are things like a shoe, a piece of cheese, or a desk; their success in selecting the self-same kingfisher is significantly lower if it occurs among pictures of other small birds (Adlam et al. 2006).

What about undergeneralization in word production and comprehension? One of the most striking instances actually comes from a contrast of these two reflections of word knowledge in a fashion that we have not yet had the opportunity to document or quantify in an experimental context but that we have observed during clinic assessments. Patients will sometimes use a specific term in talking about a particular episode involving that concept and then fail to recognize or comprehend the same term when it is presented to them in a different context. One of the patients with SD in our cohort told us that "Those trees in my garden that usually have something to eat on them didn't have anything this year because we had an early frost" (he was referring to apples). Later in the same session, when we asked him what the word "frost" means, he had no 
idea. Another patient, in describing his life history, said that he had worked as a petroleum engineer; asked later to define "petroleum," he looked puzzled, hesitated for a long time, and then hesitantly replied, "I'm not sure; is it something that uses petrol?" What is happening when a patient's comprehension fails to generalize appropriately to a term that he himself has spontaneously produced? We assume that the word can be produced when it is part of a specific scenario like apple trees in the garden or a career in petroleum engineering; the same word - when offered at a different time and in a different (or indeed without any) context - fails to make contact with that limited, scenario-specific knowledge.

A version of this undergeneralization phenomenon was documented experimentally in a clever study by Snowden et al. (1995). A patient with SD who could both produce and comprehend the term "driving license" was unable to comprehend either the more general unmodified word "license" or the compound term "dog license," even though she knew what a dog was. Over a set of such terms, the patient scored more than $90 \%$ correct in defining the personally familiar compound terms but fewer than $30 \%$ of the new compound terms, and she made no attempt to infer or guess the meanings of the latter. Making this kind of appropriate inference, even if one has never heard specifically of a dog license, is precisely the achievement of a functioning semantic memory system.

There are many anecdotal examples of undergeneralization in word production. For example, all Japanese people can tell you the name of the prefecture in which they live. One patient with SD studied by our colleague Dr. Manabu Ikeda could not produce the name of her prefecture; but she had learned, by rote memory, a list of the various prefectures in her area. If asked to name her own prefecture, she would reel off the list in order, like an overlearned poem, and stop when she got to the correct name.

\section{Object Use and Knowledge}

$\mathrm{SD}$ is an amodal disorder. Productive and expressive tasks involving words may be most sensitive at unveiling the deficits, especially early in the disease progression, but SD is never just a language disorder (Adlam et al. 2006). Therefore, patients with SD not only fail to name objects and people but also gradually, increasingly fail to know what the objects are and what to do with them and to know who the people are. How do these deficits exhibit the over- and undergeneralization that is the core topic of this chapter?
One dramatic form of overgeneralization of object knowledge can be observed in the task of delayed-copy drawing. There is relatively little point in trying to acquire information about the object knowledge of patients with SD by asking them to draw named objects: If a patient is asked, "Please draw a rhinoceros," his or her most likely response will be "What's a rhinoceros?" But patients with SD have no impairment to the visuospatial skills required to produce a drawing of a rhinoceros, as indicated by the task of giving them such a picture and asking them to copy it. The rhino stimulus picture and one direct copy of it of a patient with SD are shown in the left and middle positions in the first row of Figure 3, respectively. So far, so fairly normal. On another occasion, however, we showed this patient the same rhino picture and allowed him to look at it for several seconds to extract all the information he wanted from the stimulus; we neither asked him to name it nor offered him its name, thus making this exposure purely nonverbal. We then removed the picture and asked the patient to count from 1 to 15 , which patients with SD do without difficulty and about as rapidly as an unimpaired person, taking about 10 seconds. We then said (again without giving the picture a name), "Please draw what you were looking at a minute ago." The result is illustrated at the right-hand side of the top row of Figure 3. The rhino now has no horns, no armored skin - indeed, no rhino-specific features. It has become a generalized animal, more like a pig or a dog than a rhinoceros. Another example, this time for a peacock, is in the bottom row of FigURE 3; in the patient's delayed-copy drawing, the peacock has the four legs typical of most land animals rather than the two legs standard for birds. Many more examples of such overgeneralization in delayed-copy drawing can be found in Bozeat et al. (2003), Caine et al. (in press), Lambon Ralph and Howard (2000), and Patterson and Erzinclioglu (in press).

What is happening here? Human visual memory is not very precise or literal, at least not for more than about a second. When a normal person is asked to produce a delayed-copy drawing of a rhinoceros, the response will contain horns partly because the person remembers seeing them in the stimulus but at least as much because, having identified the picture as a rhino, the person's semantic memory will insist that the response include horns. The patient with SD cannot recognize the stimulus picture as a rhinoceros; he knows only that it is some sort of animal. After a 10second gap, short-term visual memory - which as far as we know is reasonably normal in SD - has lost substantial detail. The patient's drawing response, just like the normal individual's drawing response, will then 


\section{Stimulus picture}
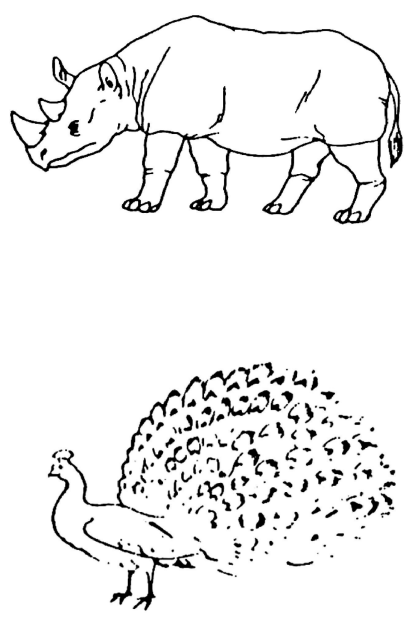

Immediate Copy
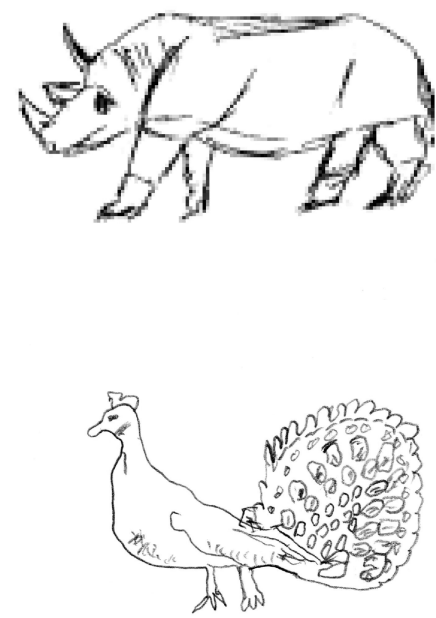

\section{Delayed copy}
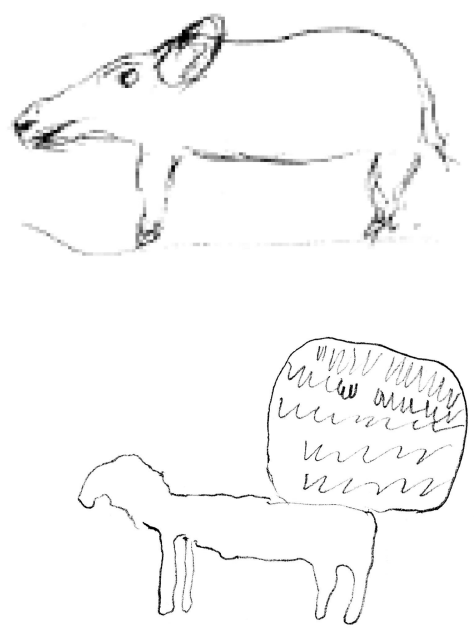

FIGURE 3. Examples of nonverbal overgeneralization: delayed-copy drawing.

be informed by conceptual knowledge of the stimulus. But for the patient, the response is degraded and overgeneralized conceptual knowledge-hence, no horns. The omission of appropriate features and the insertion of inappropriate features are anything but random in these delayed-copy drawings. Atypical features like rhinoceros horns and camel humps get omitted from the concepts where they belong and are never added to ones where they do not. Typical features like tails are rarely omitted from truly tailed animals, are often inserted onto tailless animals (like frogs and seals), and never get added to trees or chairs. As a result, the drawing response is almost invariably more typical of the stimulus class than the real stimulus was.

With respect to the overgeneralization of patients with SD of object/person recognition or object use in the real world, we have mainly anecdotal evidence to offer. For example, during a meal with one of the authors of this chapter, a patient with SD stirred sugar into his glass of wine (presumably generalizing to other drinks like tea or coffee, not to mention feeding the "sweet tooth" that many patients with FTD develop). He also used two spoons, one in each hand, to eat a plate of spaghetti, instead of the fork and knife or fork and large spoon that people who are not semantically impaired would use. With regard to overgeneralized person recognition, we have seen a woman with advanced SD emerge from the clinic room out into the public area where her husband was waiting for her, walk up to a man standing in the waiting room, and say, "I can go now"; but the recipient of this message was a stranger, not her husband. We also have anecdotal evidence of overgeneralization in everyday behavior: The wives of three different male case patients with SD in our patient cohort over the years have reported to us that their husbands continued to shave their faces much as they had done all their adult lives but also began, as the disease progressed, to shave hair on other parts of their bodies.

Undergeneralization in object use is one of the most striking aspects of the SD syndrome and brings us to the topic of how - in the context of degraded conceptual knowledge - everyday behavior comes increasingly to be dominated by current episodic and procedural experience. First documented experimentally by Snowden, Griffiths, and Neary (1994) and subsequently by Bozeat et al. (2002), patients with SD continue until late in the disease to use their own familiar objects at home but often fail to generalize such recognition and use appropriately to other exemplars of the same objects. In one example in the Bozeat et al. study, the patient J.H. could find (in its usual drawer in her kitchen) and correctly use her own cheese grater. When the experimenter offered her another grater that would, to a normal person, be equivalent, J.H. did not know what to do with it. It was furthermore clear that such "knowledge" was being maintained by regular experience. In a subsequent study (Bozeat et al. 2004), J.H.'s husband helped us to select a set of objects that his wife had recently stopped using, including the cheese grater. The 
experimenter successfully retrained her to use these, but after the training sessions ended, tests designed to probe the persistence of this regained ability demonstrated a gradual but steady decline in J.H.'s success.

We placed "knowledge" in quotation marks in the previous paragraph because there has been some debate about what exactly is maintained by regular experience (Graham et al. 1999a, 1999b; Snowden et al. 1999). Although the issue is not yet resolved, and the answer is probably not "either-or," we are inclined to think that it is more in the line of episodic, autobiographical, and/or procedural memory that is being reinforced and maintained by regular experience than it is true semantic knowledge. This may seem like slightly circular reasoning, but our conclusion is based on the fact that generalization is such a hallmark of semantic memory that, if using one's own familiar cheese grater were boosting semantic knowledge of what cheese graters are for and how they are used, this boost should generalize to a different but equally good exemplar of a cheese grater, which it tends not to do.

A similar form of undergeneralization has also been experimentally demonstrated in studies of episodic recognition memory in SD. In two studies, one of recognition memory for objects (Graham et al. 2000) and the other for famous people (Simons et al. 2001), the stimulus items were selected for each patient with SD to be either still relatively "known" or now significantly "degraded" for that patient on the basis of other tests of naming and name-picture matching. Sets of photographs of items from these known and degraded categories were presented to the patients with SD in a study phase. In the subsequent test phase of each experiment, all the studied photos plus an equal number of previously unencountered photos of objects or people were presented for yes-no recognition memory judgments ("Did you see this thing/person before in this experiment?"). In a further experimental manipulation, the photo at test was either identical to the one at study or was a different exemplar of the same concept or person. For famous people, participants might see one photo of Princess Diana at study and a different one at test; for objects, they might see a square stainless-steel toaster at study and a rectangular white plastic-bodied toaster at test. The instructions, of course, included, with many examples, the information that the participant should make "yes" responses in the test when shown different exemplars of the same person or object presented during the study phase. For normal control participants there was no significant advantage for correct "Yes, I saw this before" judgments in the same - as opposed to different - photo conditions; the same was true for the patients with SD, provided that the items came from the "known" set. There was, however, a substantial and significant drop in the number of recognition memory hits for the patients' degraded items in the different-photo condition of both experiments. In other words, generalization between different photos of Princess Diana or different toasters is supported by semantic knowledge and disabled when that knowledge deteriorates.

At least anecdotally, there is evidence that the boundaries of generalization can be shockingly reduced by degraded knowledge. One of the authors of this article when doing some extensive testing with a particular patient with SD, went to test the patient at the patient's home at 2 PM every Tuesday for several weeks. After about five of these visits, during which she always welcomed the author on his arrival at 2 PM, on one Tuesday he was unavoidably detained in arriving at her house. He stopped on the way to telephone her to explain his delay, but it was clear that she did not really understand who he was or what he was telling her. When he arrived at about 3:15, she completely failed to recognize him and, until he pulled out the standard sorts of testing materials, she was reluctant to believe that she knew him and that he was supposed to be there. With a grain of humor (but many more grains of sadness about the fate of such patients), we describe this as the "Mr. Two O'Clock" episode. This patient, like many SD cases, was an avid clockwatcher and associated the researcher's arrival with a specific time; when he arrived at a different time, he was not the same person.

Report of another dramatic occurrence of undergeneralization comes from a neurologist colleague of ours in Barcelona, Dr. Raquel Sanchez-Valle. Several patients with SD develop a passion for puzzles of one kind or another; in our experience in Britain, it is most often jigsaw puzzles, perhaps because these seem to require only elements of cognition that are well preserved in SD until late in the disease, such as good visuospatial function and good color matching. For the particular patient with SD being studied by Sanchez-Valle, however, the puzzle passion was for Sudoku. Each morning, this man would arise, put on some clothes, go out to the local news agent, buy his regular daily newspaper, return home, and complete the Sudoku puzzle; until it was finished, nothing else (such as eating breakfast) held any interest for him. Sanchez-Valle knew of the patient's fascination with Sudoku and wanted to observe him engaging in this activity, so on one of his clinic visits, she gave him a newspaper containing a Sudoku puzzle. He said (in his native Catalan), "I don't know what this is or what I should do with it." The Sudoku instructions were printed beside the puzzle, so 
Sanchez-Valle asked him to read them aloud, which he did perfectly but could not understand them and so still claimed to have no idea what to do with the puzzle. Because she had not seen him in several months, and because eventually even such nonsemantic activities and abilities do decline, she assumed that the patient was no longer doing Sudoku. Half an hour later, chatting with the patient's wife, Sanchez-Valle commented that he had apparently given up Sudoku. "What do you mean?" asked the wife in surprise. "He does them every day. In fact, I have this morning's newspaper here in which he completed the Sudoku puzzle." Sudoku exemplars in different newspapers do look slightly different; they may have different sizes or typeface fonts or background color or position on the page. It nevertheless seems almost inconceivable that someone who does Sudoku every day could fail to recognize a new "token" as belonging to that "type." We can assume only that, for this patient who was semantically impaired, Sudoku is not a token of a general type but is rather an event that belongs specifically to his morning routine of fetching the newspaper and doing the puzzle before breakfast. When a similar puzzle is produced by someone else, out of its normal context, he cannot generalize it to his everyday Sudoku experience.

As acknowledged when we first raised the question about the nature of the knowledge or memory that is maintained by everyday experience, this issue is unresolved. It seems clear from the examples given above that the concepts of frost, cheese grater, and Sudoku have severely reduced boundaries for these patients. This could be the result of a diminished semantic representation combined with specific reinforcement from a particular, repeated aspect of the meaning. In other words, just as concepts in a normal individual change and develop over time as a result of continued experience, J.H.'s concept of a grater may be not only diminished in breadth because of her disease but also strengthened in its narrow instantiation because of her regular experience (see Welbourne \& Lambon Ralph 2005 , in press, for discussion of the interaction of damage with continued learning/plasticity). Alternatively, as suggested earlier, such severely reduced boundaries could reflect the fact that when a semantic concept is sufficiently degraded, the person is forced to rely on one or more different kinds of memory or knowledge that, by their nature, do not support an appropriate level of generalization. Perhaps both explanations are partially correct. It may also be that different kinds of experience lend themselves to different degrees of reliance on these two kinds of knowledge. In the delayed-copy task, for example, we argued that literal visual mem- ory provides a poor basis for performing the task and that therefore both normal individuals and patients with SD must refer to the way in which their semantic memory processed the stimulus. The patient with SD can code the rhinoceros only as an overgeneralized animal, and his or her response will reflect that semantic coding. Regular use of a specific cheese grater, on the other hand, may be capable of producing fairly durable episodic and procedural memories that can stand in for the general concept.

A similar kind of analysis of type of task or experience might apply to the perhaps obvious question: What determines whether the abnormal boundaries for concepts in SD are too wide, hence resulting in overgeneralization, or too narrow, thus yielding undergeneralization? We have already mentioned one general principle that will contribute to this balance: Within the domain of objects or concrete concepts, a real object or picture of it gives more clues to the approximate nature of the object than its name does. Thus, presentation of an object or picture to be named or defined is more likely to result in an overgeneralization response from a patient with SD than presentation of its name, which is likely to produce "I don't know" (Lambon Ralph et al. 1999). In the domain of person recognition, we gave examples above of both overand undergeneralization in SD behavior. Again, the task or situation may in large part determine which will occur. If a patient genuinely no longer recognizes Princess Diana, then, when presented with a picture of her to name or identify, there is really no level at which overgeneralization can occur: She is just an unknown person. But when the patient with severe SD went out into the waiting room at the hospital to find her husband, she was confronted with several men to whom her profoundly impaired person recognition could overgeneralize. We realize that this is a somewhat post-hoc analysis and that we need to make more precise predictions in advance about the circumstances under which one would expect to obtain these striking phenomena of over- and undergeneralization resulting from degraded semantic memory. We are only just beginning. ...

\section{Importance of the ATLs in Semantic Memory: What Is the Evidence from Other Sources?}

Although the data from SD clearly implicate the bilateral ATL in semantic representation, these areas are often overlooked or even disputed in other research on semantic memory (Hickok \& Poeppel 2007; Martin 2007; Wise 2003). It is claimed that semantic tasks rarely activate the ATL in functional MRI (fMRI) but 
rather, in line with some aphasiological models (see below), implicate left posterior temporal, temporoparietal, and prefrontal regions (Devlin et al. 2000; Garavan et al. 2000; Martin 2007). It is also claimed that, were the ATL to be so vital to semantic memory, resection of the ATL for intractable epilepsy should result in major semantic impairment, which it does not (Hermann et al. 1999).

Recent studies indicate, however, that the results underlying these claims are not as contradictory as they might seem to the results from SD. First, the failure to find ATL activation in semantic tasks reflects, at least in part, technical limitations of fMRI. Field inhomogeneities around air-filled cavities lead to signal dropout and distortions that are particularly pronounced in the orbitofrontal cortex and the inferior and polar aspects of the temporal lobes (Devlin et al. 2000; Wise 2003). Functional neuroimaging that uses positron emission tomography (which does not suffer from the same problems) does detect semantically related activation in the ATL, even when the same experiment conducted in fMRI does not (Devlin et al. 2000). Because of the preeminence of fMRI in cognitive neuroscience, the potentially central importance of the ATL within a network of regions that support semantic cognition can be overlooked (Patterson et al. 2007; Rogers \& McClelland 2004; Wise 2003).

Second, interpretation of the cognitive consequences of epilepsy surgery is complicated by two factors: (a) Longstanding epilepsy can lead to changes in neural organization and, indeed, recent imaging studies have shown that white matter connectivity and neurotransmitter function are significantly altered in this condition (Hammers et al. 2003; Powell et al. 2007); and (b) epilepsy surgery is unilateral, whereas patients with SD invariably have bilateral, even if asymmetrical, lesions. In the rare cases where we have been able to assess patients with SD at a sufficiently early stage that there is only minor atrophy on the less affected side, the patients have tended to show rather slight semantic deficits unless taxed with especially difficult tasks. We therefore infer that substantial semantic deficit results only from significant bilateral ATL abnormality. This supposition would account for the fact that semantic deficits observed after temporal lobe resection are mild (Wilkins \& Moscovitch 1978), or indeed absent, if the tests are too easy.

Other neurological disorders, such as herpes simplex virus encephalitis, do produce semantic impairment when damage affects the same bilateral temporal lobe regions as SD (Lambon Ralph et al. 2007; Noppeney et al. 2007). Also, we have recently demonstrated that repetitive transcranial magnetic stimulation of the
ATL in normal participants produces a selective slowing of semantically related tasks (synonym judgment and naming) but not of equally demanding, nonsemantic tasks - exactly as expected from SD (Pobric et al. in press).

\section{Beyond Semantic Memory and the ATL}

The focus of this article has been the role of the ATLs in achieving conceptualization and appropriate levels of semantic generalization. We have, therefore, said nothing thus far about the role of other brain regions or different aspects of semantic cognition. There is no doubt that parts of the brain other than the ATL play critical roles in other aspects of semantically driven behavior, as evidenced by activation of these other regions in functional neuroimaging studies of semantic processing in normal participants and by some varieties of semantic disorders when these other regions are damaged. These other aspects can be divided into two broad types: impairments of semantic access and disorders of semantic control.

Most models of semantic memory assume that semantic activation is initiated by input from one or more modality-specific forms of knowledge/engrams. Sensory input must be processed well enough to enable semantic processing. Failures of comprehension or semantically driven behavior can follow, therefore, from impaired mechanisms of perceptual analysis or from damaged connections between perception and central semantic representations. Classically, these two forms of impairment are labelled "apperceptive" and "associative" agnosia (Brown 1972). The crucial characteristic of these disorders is that comprehension/semantic behavior is impaired only when input arrives in the affected modality (e.g., visual or auditory or tactile or linguistic); comprehension initiated by a stimulus in any other modality should be fairly normal. These modality-specific agnosias, or impairments of semantic access, are unlike the amodal impairment of patients with SD.

What about the other type of problem, a disorder of semantic "control"? There is recent evidence to suggest that patients with damage to non-ATL regions can exhibit multimodal semantic impairment. Some patients classified as aphasic after a left-hemisphere stroke, whose deficits might be expected to affect only language comprehension, in fact perform poorly on purely nonverbal receptive tests, such as matching of different pictures on a semantic basis, and/or on purely nonverbal production tests such as object use (especially in a complex sequence of behavior such 


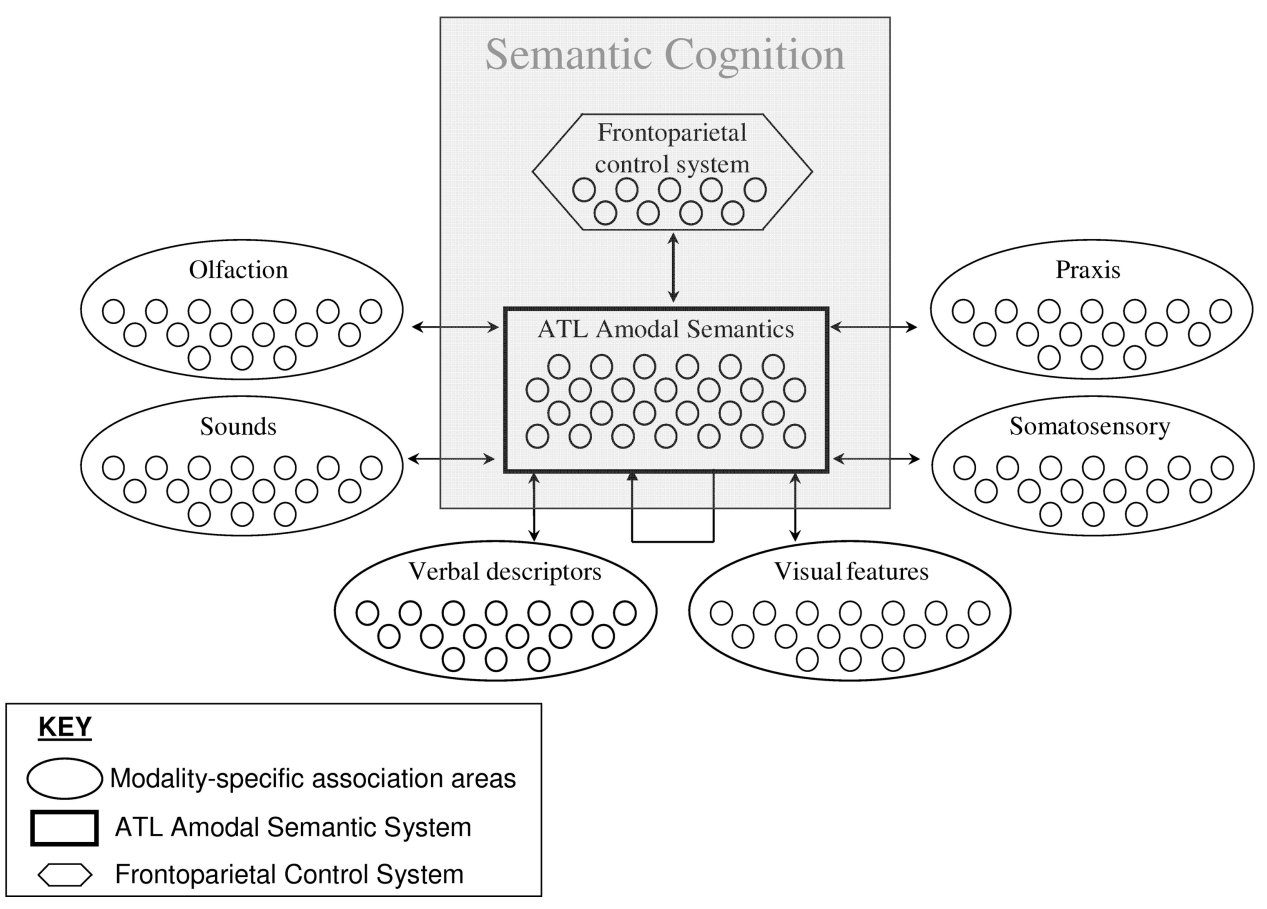

FIGURE 4. Extended theoretical framework for semantic cognition.

as making a cup of tea). These patients, described as having semantic aphasia (SA; Jefferies \& Lambon Ralph 2006), have lesions of either the left inferior frontal gyrus and/or the left temporoparietal junction (Berthier 1999). Although their multimodal semantic impairment might prompt one to think that $\mathrm{SA}$ is the stroke/aphasic equivalent of SD, direct formal comparisons between the patient groups indicate that the two disorders are qualitatively different (Jefferies \& Lambon Ralph 2006). SA seems to arise not from degraded knowledge itself but rather from an impairment of semantic control - that is, difficulty in manipulating and shaping semantic knowledge in a fashion appropriate for the specific task in hand or for the specific moment within a task. SD is a disorder of core conceptual knowledge. Because all semantic tasks (though to different degrees) require executive control as well as conceptual knowledge, both patient groups reveal deficits across both verbal and nonverbal domains. The lesion locations of the patients with SA (inferior frontal gyrus and/or temporoparietal junction) align with results from functional neuroimaging of normal subjects undertaking not only semantic tasks but also other cognitive assessments that require executive processing (Badre et al. 2005; Gold et al. 2006).

These comparative studies generate a more extensive framework (summarized in FIG. 4) about the processes and their associated brain regions that support semantically driven behavior, which we refer to as "semantic cognition" (Rogers \& McClelland 2004). We have adopted this broader term to encompass the two major, interactive dimensions that are required for flexible semantic behavior. Semantic control allows task-relevant aspects of meaning to be brought to the fore, whereas other, currently irrelevant pieces of information are inhibited, thus enabling semantic flexibility and novel semantic problem solving. As described more fully in this article semantic memory or conceptual knowledge provides the basis on which semantic information can be retrieved, and generalized at an appropriate level, irrespective of modality or task.

\section{Conflicts of Interest}

The authors declare no conflicts of interest.

\section{References}

Adlam, A.-L. R., Patterson, K., Rogers, T. T., Nestor, P., Salmond, C. H., Acosta-Cabronero, J., et al. (2006). Semantic dementia and fluent primary progressive aphasia: two sides of the same coin? Brain, 129, 3066-3080.

Badre, D., Poldrack, R., Paré-Blagoev, E., Insler, R., \& Wagner, A. (2005). Dissociable controlled retrieval and generalized selection mechanisms in ventrolateral prefrontal cortex. Neuron, 47, 907-918. 
Berthier, M. (1999). Transcortical Aphasias. Hove, East Sussex: Psychology Press.

Bozeat, S., Lambon Ralph, M. A., Graham, K. S., Patterson, K., Wilkin, H., Rowland, J., et al. (2003). A duck with four legs: investigating the structure of conceptual knowledge using picture drawing in semantic dementia. Cognitive Neuropsychology, 20, 27-47.

Bozeat, S., Lambon Ralph, M. A., Patterson, K., \& Hodges, J. R. (2002). The influence of personal familiarity and context on object use in semantic dementia. Neurocase, $8,127-$ 134.

Bozeat, S., Patterson, K., \& Hodges, J. R. (2004). Relearning object use in semantic dementia. Neuropsychological Rehabilitation, 14, 351-363.

Brambati, S. M., Rankin, K. P., Narvid, J., et al. (in press). 2007. Atrophy progression in semantic dementia with asymmetric temporal involvement: a tensor-based morphometry study. Neurobiol. Aging. [Epub ahead of print: doi:10.1016/j.neurobiolaging.2007.05.014]

Brown, J. W. (1972). Aphasia, Apraxia and Agnosia. Springfield, IL: Charles C Thomas.

Caine, D., Breen, N., \& Patterson, K. (2007). Emergence and progression of "non-semantic" deficits in semantic dementia. Cortex, in press.

Collins, A. M., \& Quillian, M. R. (1969). Retrieval time from semantic memory. Fournal of Verbal Learning and Verbal Behavior, $8,240-247$.

Damasio, A. R. (1989). The brain binds entities and events by multiregional activation from convergence zones. Neural Computation, 1, 123-132.

Desgranges, B. et al. (2007) Anatomical and functional alterations in semantic dementia: a voxel-based MRI and PET study. Neurobiology of Aging, 28, 1904-1913.

Devlin, J. T., Russell, R. P., Davis, M. H., Price, C. J., Wilson, J., Moss, H. E., et al. (2000). Susceptibility-induced loss of signal: comparing PET and fMRI on a semantic task. Neuroimage, 11, 589-600.

Diehl, B. et al. (2004). Cerebral metabolic patterns at early stages of frontotemporal dementia and semantic dementia. A PET study. Neurobiology of Aging, 25, 1051-1056.

Eggert, G. H. (1977). Wernicke's Works on Aphasia: A Sourcebook and Review (Vol.1). The Hague: Mouton.

Garavan, H., Ross, T.J., Li, S. J., \& Stein, E. A. (2000). A parametric manipulation of central executive functioning. Cerebral Cortex, 10, 585-592.

Garrard, P., Lambon Ralph, M. A., Hodges, J. R., \& Patterson, K. (2001). Prototypicality, distinctiveness, and intercorrelation: analyses of the semantic attributes of living and nonliving concepts. Cognitive Neuropsychology, 18, 125-174.

Gloor, P. (1997). The Temporal Lobe and the Limbic System. Oxford, UK: Oxford University Press.

Gold, B. T. et al. (2006). Dissociation of automatic and strategic lexical-semantics: functional magnetic resonance imaging evidence for differing roles of multiple frontotemporal regions. Fournal of Neuroscience, 26, 6523-6532.

Goodglass, H., \& Kaplan, E. (1983). The Assessment of Aphasia and Related Disorders. 2nd ed. Philadelphia: Lea \& Febiger.

Graham, K. S., Lambon Ralph, M. A., \& Hodges, J. R. (1999a). Determining the impact of autobiographical experience on "meaning": new insights from investigating sports- related vocabulary and knowledge in two cases of semantic dementia. Cognitive Neuropsychology, 14, 801-837.

Graham, K. S., Lambon Ralph, M. A., \& Hodges, J. R. (1999b). A questionable semantics: the interaction between semantic knowledge and autobiographical experience in semantic dementia. Cognitive Neuropsychology, 16, 689-698.

Graham, K. S., Patterson, K., \& Hodges, J. R. (1995). Progressive pure anomia: insufficient activation of phonology by meaning. Neurocase, 1, 25-38.

Graham, K. S., Simons, J. S., Pratt, K. H., Patterson, K., \& Hodges, J. R. (2000). Insights from semantic dementia on the relationship between episodic and semantic memory. Neuropsychologia, 38, 313-324.

Hammers, A., Koepp, M. J., Richardson, M. P., Hurlemann, R., Brooks, D. J., \& Duncan, J. S. (2003). Grey and white matter flumazenil binding in neocortical epilepsy with normal MRI. A PET study of 44 patients. Brain, 126, 1300-1318.

Hermann, B., Davies, K., Foley, K., \& Bell, B. (1999). Visual confrontation naming outcome after standard left anterior temporal lobectomy with sparing versus resection of the superior temporal gyrus: a randomized prospective clinical trial. Epilepsia, 40, 1070-1076.

Hickok, G., \& Poeppel, D. (2007). Opinion - the cortical organization of speech processing. Nature Reviewes Neuroscience, 8 , 393-402.

Hodges, J. R., Graham, N., \& Patterson, K. (1995). Charting the progression in semantic dementia: implications for the organisation of semantic memory. Memory, 3, 463495.

Hodges, J. R., \& Patterson, K. (2007). The neuropsychology of frontotemporal dementia. In J. R. Hodges (Ed.), Frontotemporal Dementia Syndromes (pp. 102-133). Cambridge, UK: Cambridge University Press.

Hodges, J. R., Patterson, K., Oxbury, S., \& Funnell, E. (1992). Semantic dementia: progressive fluent aphasia with temporal lobe atrophy. Brain, 115, 1783-1806.

Jefferies, E., \& Lambon Ralph, M. A. (2006). Semantic impairment in stroke aphasia vs. semantic dementia: a case-series comparison. Brain, 129, 2132-2147.

Kipps, C. M., Knibb, J. A., Patterson, K., \& Hodges, J. H. (2008). Neuropsychology of frontotemporal dementia. In G. Goldenberg \& B. Miller (Eds.), Handbook of Clinical Neurology. Amsterdam: Elsevier.

Lambon Ralph, M. A., Graham, K. S., Patterson, K., \& Hodges, J. R. (1999). Is a picture worth a thousand words? Evidence from concept definitions by patients with semantic dementia. Brain \& Language, 70, 309-335.

Lambon Ralph, M. A., \& Howard, D. (2000). Gogi aphasia or semantic dementia? Assessing poor verbal comprehension in a case of fluent progressive aphasia. Cognitive Neuropsychology, 17, 437-466.

Lambon Ralph, M. A., Lowe, C., \& Rogers, T. T. (2007). Both type and distribution of damage are critical for categoryspecific semantic deficits: evidence from semantic dementia, herpes simplex virus encephalitis and a neural network model of conceptual knowledge. Brain, 130, 1127 1137.

Martin, A. (2007). The representation of object concepts in the brain. Annual Review of Psychology, 58, 25-45. 
Morrow, L. I., \& Duffy, F. M. (2005). The representation of ontological category concepts as affected by healthy aging: normative data and theoretical implications. Behavior Research Methods, 37, 608-625.

Nestor, P. J., Fryer, T. D., \& Hodges, J. R. (2006). Declarative memory impairments in Alzheimer's disease and semantic dementia. NeuroImage, 30, 1010-1020.

Noppeney, U., Patterson, K., Tyler, L. K., Moss, H., Stamatakis, E. A., Bright, P., et al. (2007). Temporal lobe lesions and semantic impairment: a comparison of herpes simplex virus encephalitis and semantic dementia. Brain, 130, 11381147.

Patterson, K. (2007). The reign of typicality in semantic memory. Philosophical Transactions of the Royal Society B, 362, 813-821.

Patterson, K., \& Erzinçlioğlu, S. (in press). Drawing as a "window" on deteriorating conceptual knowledge in neurodegenerative disease. In C. Lange-Kuettner \& A. Vinter (Eds.), Drawing and the Non-Verbal Mind. A Life-Span Perspective. Cambridge, UK: Cambridge University Press.

Patterson, K., Graham, N. L., Lambon Ralph, M. A., \& Hodges, J. R. (2008). Varieties of silence: the impact of neurodegenerative diseases on language systems in the brain. In J. R. Pomerantz (Ed.), Topics in Integrative Neuroscience: From Cells to Cognition (pp. 181-205). Cambridge, UK: Cambridge University Press.

Patterson, K., \& Hodges, J. R. (2000). Semantic dementia: one window on the structure and organization of semantic memory. In L. Cermak (Ed.), Handbook of Neuropsychology, 2nd Edition, Vol. 2: Memory and Its Disorders (pp. 313-333). Amsterdam/London: Elsevier.

Patterson, K., \& MacDonald, M. C. (2006). Sweet nothings: Narrative speech in semantic dementia. In S. Andrews (Ed.), From Inkmarks to Ideas: Current Issues in Lexical Processing (pp. 288-317). Hove, UK: Psychology Press.

Patterson, K., Nestor, P. J., \& Rogers, T. T. (2007). Where do you know what you know? the representation of semantic knowledge in the human brain. Nature Reviewes Neuroscience, 8, 976-987.

Pobric, G., Jefferies, E., \& Lambon Ralph, M. A. (2007). Anterior temporal lobes mediate semantic representation: mimicking semantic dementia by using rTMS in normal participants. Proceedings of the National Academy of Sciences USA, 104, 20137-20141.

Powell, H. W. R., Parker, G. J. M., Alexander, D. C., Symms, M. R., Boulby, P. A., Wheeler-Kingshott, C. A. M., et al. (2007). Abnormalities of language networks in temporal lobe epilepsy. Neuroimage, 36, 209-221.

Quillian, M. R. (1966). Semantic Memory. Ph.D. thesis, Carnegie Institute of Technology.

Rogers, T. T., Lambon Ralph, M. A., Garrard, P., Bozeat, S., McClelland, J. L., Hodges, J.R., et al. (2004). The structure and deterioration of semantic memory: a neuropsychological and computational investigation. Psychological Review, 111, 205-235.
Rogers, T. T., \& McClelland, J. L. (2004). Semantic Cognition: A Parallel Distributed Processing Approach. Cambridge, MA: MIT Press.

Shallice, T. (1988). From Neuropsychology to Mental Structure. Cambridge, UK: Cambridge University Press.

Simons, J. S., Graham, K. S., Galton, C. J., Patterson, K., \& Hodges, J. R. (2001). Semantic knowledge and episodic memory for faces in semantic dementia. Neuropsychology, $15,101-114$.

Snowden, J. S., Goulding, P. J., \& Neary, D. (1989). Semantic dementia: a form of circumscribed cerebral atrophy. Behavioural Neurology, 2, 111-138.

Snowden, J. S., Griffiths, H. L., \& Neary, D. (1994a). Semantic dementia: autobiographical contribution to preservation of meaning. Cognitive Neuropsychology, 11, 265-288.

Snowden, J. S., Griffiths, H. L., \& Neary, D. (1995). Autobiographical experience and word meaning. Memory, 3, 225246.

Snowden, J. S., Griffiths, H. L., \& Neary, D. (1999). The impact of autobiographical experience on meaning: reply to Graham, Lambon Ralph and Hodges. Cognitive Neuropsychology, 16, 673-687.

Snowden, J. S., Neary, D., \& Mann, D. M. A. (1996). FrontoTemporal Lobar Degeneration. New York: Churchill Livingstone.

Thompson-Schill, S. L. (2003). Neuroimaging studies of semantic memory: inferring "how" from "where." Neuropsychologia, 41(3), 280-292.

Tulving, E. (1972). Episodic and semantic memory. In E. Tulving \& W. Donaldson (Eds.), Organisation of Memory (pp. 381403). New York: Academic Press.

Warrington, E. K. (1975). The selective impairment of semantic memory. Quarterly Fournal of Experimental Psychology, 27, 635-657.

Welbourne, S. R., \& Lambon Ralph, M. A. (2005). Exploring the impact of plasticity-related recovery after brain damage in a connectionist model of single word reading. Cognitive, Affective \& Behavioral Neuroscience, 5, 77-92.

Welbourne, S. R., \& Lambon Ralph, M. A. (2007). Using PDP models to simulate phonological dyslexia: the key role of plasticity-related recovery. Fournal of Cognitive Neuroscience, 19, 1125-1139.

Wilkins, A. J., \& Moscovitch, M. (1978). Selective impairment of semantic memory after temporal lobectomy. Neuropsychologia, 16, 73-79.

Williams, G. B., Nestor, P. J., \& Hodges, J. R. (2005). Neural correlates of semantic and behavioural deficits in frontotemporal dementia. Neuroimage, 24, 1042-1051.

Wise, R. (2003). Language systems in normal and aphasic human subjects: functional imaging studies and inferences from animal studies. British Medical Bulletin, 65, 95-119.

Woollams, A. M., Cooper-Pye, E., Hodges, J. R., \& Patterson, K. (2007 revision). Anomia: a doubly typical signature of semantic dementia. Manuscript under revision. 